\title{
Software engineering management
}

\author{
Mauricio Rojas Contreras, Msc. ${ }^{1}$, Maritza Sánchez Delgado, Msc. ${ }^{2}$ \\ ${ }^{1}$ Universidad de Pamplona, Pamplona, Norte de Santander, Colombia, mrojas@unipamplona.edu.co \\ ${ }^{2}$ Universidad de Pamplona, Pamplona, Norte de Santander, Colombia, mpilas@unipamplona.edu.co
}

\begin{abstract}
The management of software engineering can be defined as the application of management activities to the software engineering process conceived as the set of technical and management activities within the life cycle processes of the software. From this point of view, this article describes a method to develop software engineering management activities in order to eliminate the common perception that the software industry delivers its products late, increasing the budget of the projects above the planned and the lack of precision in the implementation of the project requirements. The result of the work is the specification of a method conceived as a set of steps to make software engineering management, which brings as an added value the increase in the accuracy in the process of verifying the estimates of time, costs and scope with respect to to what was observed in the execution of the software project.

Keywords- Management Software engineering, Project management, software engineering process
\end{abstract}




\title{
Gestión de Ingeniería del software
}

\author{
Mauricio Rojas Contreras, Msc. ${ }^{1}$, Maritza Sánchez Delgado, Msc. ${ }^{2}$ \\ ${ }^{1}$ Universidad de Pamplona, Pamplona, Norte de Santander, Colombia,mrojas@unipamplona.edu.co \\ ${ }^{2}$ Universidad de Pamplona, Pamplona, Norte de Santander, Colombia, mpilas@ unipamplona.edu.co
}

\begin{abstract}
La gestión de la ingeniería del software se puede definir como la aplicación de actividades de gestión al proceso de ingeniería del software concebido como el conjunto de actividades técnicas y de gestión dentro de los procesos de ciclo de vida del software. Desde este punto de vista, el presente artículo describe un método para desarrollar las actividades de gestión de Ingeniería del software con el fin de eliminar la percepción común de que la industria del software entrega sus productos tarde, incrementando el presupuesto de los proyectos por encima de lo planeado y la falta de precisión en la implementación de los requerimientos del proyecto. El resultado del trabajo es la especificación de un método concebido como un conjunto de pasos para hacer gestión de ingeniería del software, lo cual trae como valor agregado el aumento en la precisión en el proceso de verificar las estimaciones de tiempo, costos y alcances con respecto a lo observado en la ejecución del proyecto software.
\end{abstract}

Keywords-Gestión Ingeniería del software, Gestión de proyectos, proceso ingeniería software

\section{INTRODUCCION}

Los proyectos de desarrollo de software están compuestos de actividades que pueden ser clasificadas en dos grupos: actividades de gestión y actividades técnicas. Las actividades de gestión están directamente relacionadas con procesos de dirección de proyectos utilizados dentro del proyecto, mientras que las actividades técnicas se refieren a aquellas propias del desarrollo del producto software y que están determinadas por los modelos de proceso de software. [1]

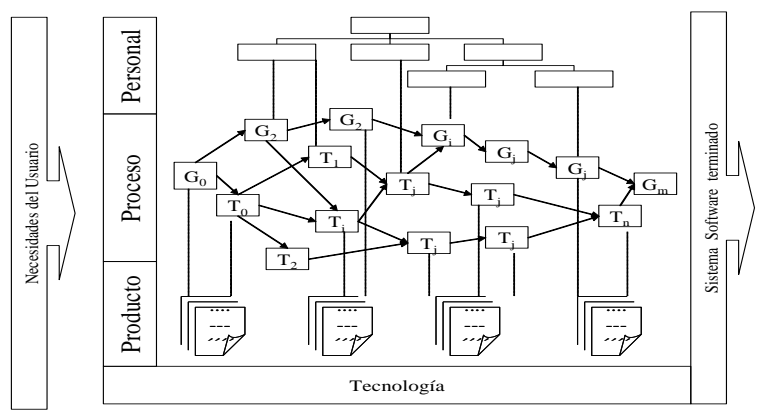

Fig. 1 Componentes de un proyecto software

En un sentido más amplio, la gestión de la ingeniería del software tiene como alcance la gestión y la medición de la Ingeniería del software. Desde el punto de vista de la gestión se utiliza el cuerpo de conocimiento de la dirección de proyectos (PMBOK®) como soporte conceptual para la especificación del método propuesto de gestión de Ingeniería del software. El PMBOK® incluye las siguientes áreas de conocimiento: gestión de integración del proyecto, gestión de alcance, gestión de tiempos, gestión de costos, gestión de calidad, gestión de recursos, gestión de comunicaciones, gestión de adquisiciones y gestión de interesados en el proyecto. Cada una de estas áreas está estructurada en procesos, los cuales encapsulan actividades que se deben desarrollar para asegurar que los procesos se desarrollan de acuerdo a las políticas organizacionales. De forma complementaria, el PMBOK® define otra vista denominada de grupos de proceso, en la cual define los siguientes: Grupo de procesos de inicio, grupo de procesos de planeación, grupo de procesos de ejecución, grupo de procesos de seguimiento y control, grupo de procesos de cierre. Los anteriores grupos de procesos están articulados con las subáreas de conocimiento de la gestión de la ingeniería del software identificadas en el cuerpo de conocimiento de la ingeniería del software. [2]

Este trabajo tiene como motivación la especificación de un método de gestión de ingeniería del software basado en el cuerpo de conocimiento de la gestión de proyectos y el cuerpo de conocimiento de la Ingeniería del software con el valor agregado de mejorar la precisión en el proceso de estimación de tiempos, costos y alcances en proyectos de software.

El artículo está organizado de la siguiente manera: En la sección 2 , se presenta una visión general de la gestión de ingeniería del software; en la sección 3 se especifica el conjunto de actividades de gestión que estructuran el método; en la sección 4 se describe el proceso de verificación y resultados de la aplicación del método en un experimento de desarrollo de un proyecto de software; finalmente, en la sección 5, se presentan las conclusiones del trabajo.

\section{GESTION DE LA INGENIERIA DEL SOFTWARE}

La gestión de la ingeniería del software puede definirse como la aplicación de actividades de gestión que asegure un desarrollo y mantenimiento del software sistemático, disciplinado y cuantificado. [3]

El cuerpo de conocimiento de la ingeniería del software estructura el área de gestión de la ingeniería del software en las siguientes subáreas: definición de inicio y alcance, planeación del proyecto software, Ejecución del proyecto software, seguimiento y control del proyecto, cierre del proyecto. [4] En forma complementaria, el cuerpo del conocimiento de la dirección de proyectos agrupa los procesos en cinco categorías conocidas como Grupos de Procesos de la Dirección de Proyectos (o Grupos de Procesos): 
Grupo de Procesos de Inicio. Aquellos procesos realizados para definir un nuevo proyecto o nueva fase de un proyecto existente al obtener la autorización para iniciar el proyecto o fase.

Grupo de Procesos de Planificación. Aquellos procesos requeridos para establecer el alcance del proyecto, refinar los objetivos y definir el curso de acción requerido para alcanzar los objetivos propuestos del proyecto.

Grupo de Procesos de Ejecución. Aquellos procesos realizados para completar el trabajo definido en el plan para la dirección del proyecto a fin de satisfacer las especificaciones del mismo.

Grupo de Procesos de Seguimiento y Control. Aquellos procesos requeridos para rastrear, revisar y regular el progreso y el desempeño del proyecto, para identificar áreas en las que el plan requiera cambios y para iniciar los cambios correspondientes.

Grupo de Procesos de Cierre. Aquellos procesos realizados para finalizar todas las actividades a través de todos los Grupos de Procesos, a fin de cerrar formalmente el proyecto o una fase del mismo. [2]

En la figura 2 se puede observar la articulación de los grupos de procesos de la gestión de proyectos con las actividades propias del proceso de desarrollo de software.

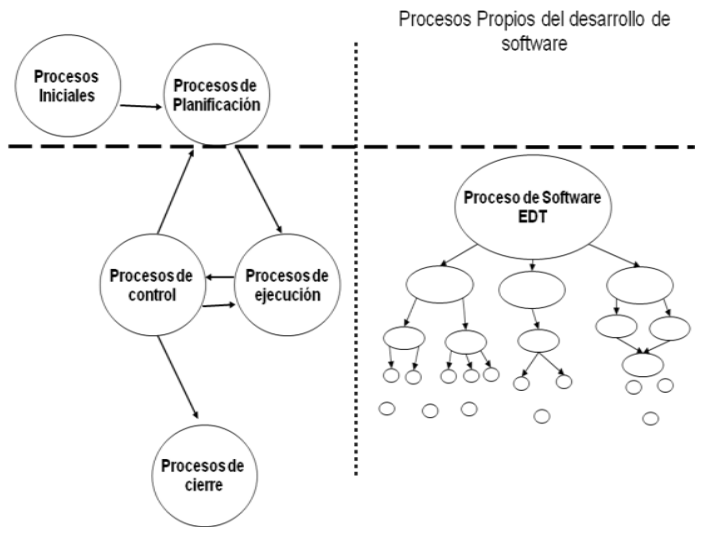

Fig. 2 Grupo de procesos de gestión del proyecto vs procesos de desarrollo [5]

Desde el punto de vista conceptual se puede verificar que las subareas de la gestión de la Ingeniería del software del cuerpo del conocimiento de la Ingeniería del software están soportadas y guardan correspondencia con los grupos de procesos del cuerpo de conocimiento de la gestión del proyecto.

\section{ESPECIFICACION DEL METODO DE GESTION DE LA INGENIERIA DEL SOFTWARE}

Los proyectos de software deben ser gestionados con el fin de responder adecuadamente a los indicadores de alcance, tiempos y costos, para lo cual se presenta la especificación de un método de gestión de ingeniería de software soportado en los grupos de proceso del PMBOK®. La estructura del método se presenta a continuación.

Para la especificación del método de gestión de Ingeniería del software se debe iniciar con la representación de la estructura general de división del trabajo del proyecto software en la cual se representan dos grupos de actividades generales: Las actividades de gestión del proyecto software y las actividades propias del proceso de desarrollo de software de la siguiente manera.

\section{PROYECTO SOFTWARE}

Actividades de gestión de gestión de proyectos

Actividades de Proceso de desarrollo de software

Posteriormente a través de método de descomposición se procede a identificar los subgrupos de procesos de gestión de la siguiente forma:

\section{PROYECTO SOFTWARE}

Actividades de gestión de proyectos

Actividades de Inicio

Actividades de Planeación

Actividades de Ejecución

Actividades de Seguimiento y control

Actividades de cierre

Actividades de Proceso de desarrollo de software

Posteriormente, a través del método de descomposición se identifican las subactividades de cada uno de los grupos de actividades del nivel anterior de la siguiente forma:

\section{PROYECTO SOFTWARE}

\section{Actividades de gestión de proyectos}

Actividades de Inicio

Construir el acta de constitución del proyecto

Identificación de stakeholders (interesados)

\section{Actividades de Planeación}

Elaborar el plan de gestión del proyecto

Elaborar el plan de gestión de alcance

Identificar requisitos del proyecto

Definir el alcance del proyecto

Crear la Estructura de división del trabajo (EDT)

Elaborar el plan de gestión de tiempos Identificar las actividades del proyecto Secuenciar las actividades del proyecto 
Estimar los tiempos de las actividades

Estimar los recursos de las actividades

Elaborar el cronograma

Elaborar el plan de gestión de costos

Estimar los costos del proyecto

Determinar el presupuesto del proyecto

Elaborar el plan de gestión de calidad

Elaborar el plan de gestión de recursos

Elaborar el plan de gestión de las comunicaciones

Elaborar el plan de gestión de riesgos

Identificar los riesgos

Desarrollar el análisis cuantitativo de riesgos

Desarrollar el análisis cualitativo de riesgos

Elaborar el plan de respuesta a los riesgos

Elaborar el plan de gestión de las adquisiciones

Elaborar el plan de gestión de stakeholders

Actividades de Ejecución

Dirigir y gestionar el trabajo del proyecto

Actividades del director de proyecto durante

la elaboración del producto

Realizar el aseguramiento de la calidad

Adquirir el equipo del proyecto

Actividades previstas para la incorporación de personal del proyecto previstas en el plan de gestión de recurso humano

Gestionar convocatorias

Gestionar entrevistas

Seleccionar personal

Desarrollar el equipo del proyecto

Actividades previstas para la capacitación de personal del proyecto previstas en el plan de gestión de recurso humano

Desarrollar cursos

Gestionar reuniones de capacitación

Dirigir el equipo del proyecto

Actividades previstas para la integración y motivación del personal del proyecto previstas en el plan de gestión de recurso humano

Gestionar y desarrollar reuniones de integración

Gestionar y desarrollar charlas de motivación

Dirigir las comunicaciones

Actividades previstas en el plan de gestión de comunicaciones

Desarrollar Reuniones

Envío de reportes y comunicaciones

Gestionar la participación de los interesados
Actividades previstas en el plan de gestión de stakeholders

Reuniones con stakeholders

Entrevistas con stakeholders

Efectuar las Adquisiciones

Actividades previstas en el plan de gestión de adquisiciones

Ejecutar compras

Recepción de materiales adquiridos

Recepción de equipos adquiridos

Recepción de servicios contratados

Actividades de seguimiento y control

Monitorear y controlar el trabajo del proyecto

Actividades periódicas relacionadas con el seguimiento al desarrollo de las actividades técnicas del proyecto

Ejecutar Auditorias

Análisis de informes de avance

Realizar Control integrado de Cambios

Actividades relacionadas con la recepción y aprobación de solicitudes de cambios en los requerimientos del proyecto.

Actividades relacionadas con los procedimientos para la atención a solicitudes de cambios.

Validar el alcance

Actividades de validación previstas en el plan de gestión de alcance

Actividades relacionadas con la formalización de la aceptación de los entregables

Auditorías a entregables

Encuestas de satisfacción de entregables

Inspecciones de alcance

Elaboración de actas de entrega

Controlar el Alcance

Actividades relacionadas con los cambios de alcance solicitados

Análisis de variación respecto a las solicitudes de cambio de alcance

Controlar el Cronograma

Actividades relacionadas con el monitoreo de fechas según el plan de gestión de cronograma

Recepción de solicitudes y aprobación de cambios relacionados con el cronograma Revisiones del desempeño

Controlar los costos

Actividades de seguimiento a los gastos de acuerdo al plan de gestión de costos

Atención de solicitudes de cambio de costos

Controlar la Calidad

Actividades previstas en el plan de gestión de calidad

16 $^{\text {th }}$ LACCEI International Multi-Conference for Engineering, Education, and Technology: "Innovation in Education and 


\author{
Proceso de medición \\ Procesos estadísticos de control de calidad \\ Controlar las comunicaciones \\ Actividades previstas en el plan de gestión de \\ comunicaciones relacionadas con asegurar \\ que se satisfagan las necesidades de \\ información de los miembros del equipo y \\ demás interesados \\ Reuniones \\ Envío de informes \\ Controlar los riesgos \\ Reevaluación periódica de riesgos \\ Identificación de nuevos riesgos \\ Auditoria de riesgos \\ Análisis de variación y de tendencias de \\ riesgos \\ Reuniones relacionadas con el monitoreo y \\ control de riesgos \\ Controlar las adquisiciones \\ Actividades previstas en el plan de gestión de \\ adquisiciones relacionadas con el control de \\ proveedores \\ Revisión periódica de contratos de \\ adquisiciones \\ Auditorías a los procesos de compras del \\ proyecto. \\ Controlar la participación de los interesados \\ Actividades previstas en el plan de gestión de \\ interesados \\ Monitorear la relación con los stakeholders \\ Ajustar las estrategias de gestión de \\ stakeholders \\ Actividades de cierre \\ Cerrar el Proyecto \\ Cerrar las adquisiciones
}

La organización de actividades descrita se puede considerar como la estructura de división de trabajo (EDT) ideal para un proyecto de software en lo referente a las actividades de gestión de proyecto, las actividades se pueden ajustar de acuerdo a las necesidades y requerimientos del proyecto.

El método utiliza la estrategia de descomposición para especificar las actividades técnicas del proyecto dependiendo del modelo de proceso de desarrollo empleado, en el caso particular del método se utiliza un modelo en cascada, obteniendo como resultado una organización de actividades del proceso de desarrollo de software con la estructura siguiente:

\section{PROYECTO SOFTWARE}

Actividades de gestión de gestión de proyectos

Actividades de Proceso de desarrollo de software
Actividades de modelamiento del negocio

Identificación de procesos de negocio

Identificación de procesos de soporte

Construcción del modelo del negocio

Actividades de requerimientos

Identificación de requerimientos funcionales

Identificación de requerimientos no funcionales

Construcción del documento de especificación del sistema

\section{Actividades de Análisis}

Construcción del modelo funcional

Construcción del modelo de clases y objetos

Construcción del modelo dinámico

Estructurar el modelo de análisis

Actividades de Diseño

Identificación de subsistemas

Definición de la arquitectura de software

Identificación de los objetivos de diseño

Actividades de Implementación e integración

Implementación del subsistema 1

Implementación del subsistema .. n

Integración de subsistemas

\section{Actividades de pruebas}

Aplicación de pruebas unitarias

Aplicación de pruebas de integración

Aplicación de pruebas del sistema

La especificación del método de gestión de la Ingeniería del software se estructura a través de la integración de la estructura de actividades de gestión de proyectos con las actividades técnicas del proceso de desarrollo de software.

\section{RESULTADOS DE LA VALIDACION DEL METODO}

El método presentado fue validado a través de la toma de valores a un grupo control sin ningún método de gestión de la ingeniería del software y con un grupo experimental que utilizo un segmento del método de gestión de Ingeniería del software para verificar los valores definidos en el proceso de estimación de tiempos y alcance. Se debe aclarar que el grupo experimental recibió capacitación en el alcance y estructura del método propuesto. Para llevar a cabo el experimento se definió un proyecto tipo con 6 funcionalidades, el proyecto fue gestionado y desarrollado por el grupo control y el grupo experimental. Los resultados de la comparación de valores del grupo control y el grupo experimental se observan en la figura 3. 


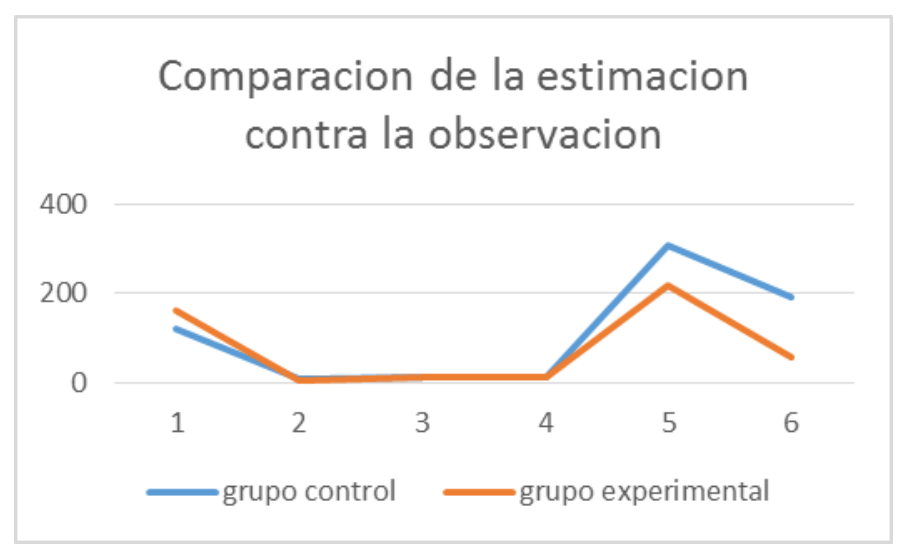

Fig. 3 Comparativo de valores del grupo control y el grupo experimental.

De acuerdo al experimento se evidencio que con la aplicación del método en el grupo experimental se mejoraron los indicadores de precisión en la estimación de tiempos del proyecto tipo, en forma específica se pudo determinar que el grupo experimental obtuvo un $345 \%$ de mejora en la estimación de tiempos total del proyecto utilizando un método como el descrito en este trabajo.

\section{CONCLUSIONES}

Los proyectos de desarrollo de software están compuestos estructuralmente por actividades de gestión y actividades de técnicas derivadas del proceso de desarrollo de software utilizado. Las actividades técnicas normalmente están asociadas con los modelos de desarrollo de software, sin embargo, solo algunas metodologías abordan de forma general la especificación de las actividades de gestión que estructuran el área de conocimiento de la Gestión de la Ingeniería del software.

La ausencia de la especificación de un conjunto de actividades de gestión en proyectos de software potencialmente puede materializar riesgos cono el aumento de costos en los proyectos, aumento de tiempos y potencialmente se pueden variar los alcances del proyecto lo cual genera una disminución en los indicadores de calidad del proyecto.

La especificación del método de gestión de Ingeniería del software puede variar en la aplicación de acuerdo a las necesidades o problemas a solucionar por el proyecto software, sin embargo independientemente de la metodología de desarrollo o de las actividades de gestión siempre habrá actividades y productos comunes especificados en el método propuesto.

El uso de métodos de gestión de Ingeniería del software permite mejorar los indicadores de precisión en la estimación de tiempos, costos y alcances, lo cual trae como consecuencia el incremento en los indicadores de calidad del proyecto.
El diseño del método propuesto tiene como principio la utilización de la técnica de descomposición de las actividades en subactividades granulares, con el fin de disminuir la complejidad del proyecto, aumentar la precisión y facilitar los procesos de estimación. Adicionalmente, esta técnica permite que cada grupo de actividades sea ejecutado por un grupo pequeño de personas.

El cuerpo de conocimiento de la gestión de proyectos y el cuerpo de conocimiento de la Ingeniería del software se convierten en forma integrada en base conceptual para solucionar problemas de la Ingeniería del software, en forma particular del área de conocimiento de la Gestión de la Ingeniería del software al proponer una especificación de método que subsane la ausencia de una guía especifica que describa las actividades de gestión que se deben desarrollar en un proyecto software.

\section{RECONOCIMIENTO}

Este trabajo se ha desarrollado con la colaboración de la Universidad de Pamplona a través del grupo de investigación Ciencias Computacionales "CICOM" y la Maestría en Gestión de Proyectos Informáticos.

\section{REFERENCIAS}

[1] M. Rojas, M. Sanchez and L. Esteban, "Planeacion de proyectos de software basado en el PMBOK®" LACCEI 2014.

[2] SWEBOK (2004). Guide to the Software Engineering Body of Knowledge. SWEBOK 2004 Version. A project of the IEEE Computer Society Professional Practices Comité.

[3] IEEE Computer Society. (2004). Guide to the Software Engineering Body of Knowledge, A project of the IEEE Computer Society Professional Practices Committee. Recuperado de: http://www.math.unipd.it/ tullio/IS1/2007/Approfondimenti/SWEBOK.pdf

[4] A guide to the Project Management Body of Knowledge 4 Edition, PMI, 2008.

[5] M. Rojas, M. Sanchez and L. Esteban, "Planeacion de proyectos de software basado en el PMBOK®” LACCEI 2014.

[6] Boehm, B., The Spiral model as a tool for evolutionary software acquisition, CrossTalk, mayo 2001, disponible en www.stcs.hill.af.mil/crosstalk/2001/05/boehm.html.B. Simpson, et al, "Title of paper goes here if known," unpublished.

[7] B. Bruegge; A. Dutoit. Ingeniería de software orientado a objetos. Editorial Pearson. México. 2002.

[8] G. Pantaleo, L, Rinaudo, "Ingenieria del software". Editorial Alfaomega, Buenos Aires, 2105.

[9] Bucanac, C., The V-model. University of Karlsklona/Ronneby, enero de 1999. www.bucanac.com/documents/The_V-model.pdf

[10] V, Garcia.; J. Salvarredy; J. García. Project Management Utilizando Microsoft Visio y Microsoft Project. Editorial Omicron. Buenos Aires. 2007.

[11] Pressman, R. Ingeniería del software: un enfoque práctico. Séptima edición. 2010.

[12] M. Rojas, M. Sanchez, Gestión de alcance en proyectos de software basados en modelos de procesos lineales. LACCEI 2017 